Mon. Not. R. Astron. Soc. 000, 1-8 (2009) Printed 9 November $2018 \quad$ (MN LATEX style file v2.2)

\title{
The X-ray Binary Analogy to the First AGN QPO
}

\author{
Matthew Middleton ${ }^{1}$, Chris Done ${ }^{1}$ \\ ${ }^{1}$ Department of Physics, University of Durham, South Road, Durham DH1 3LE, UK
}

9 November 2018

\begin{abstract}
The Narrow Line Seyfert 1 galaxy REJ1034+396 is so far unique amongst AGN in showing a Quasi-periodic oscillation (QPO) in its variability power spectrum. There are multiple types of QPO seen in black hole binary (BHB) systems, so we need to identify which BHB QPO corresponds to the one seen in the AGN. A key hint is the 'hot disc dominated' energy spectrum of REJ1034+396 which is sufficiently unusual that it suggests a mildly super-Eddington flow, also favoured by the most recent mass estimates for the AGN. This suggests the $67 \mathrm{~Hz}$ QPO seen occasionally in the mildly super-Eddington BHB GRS 1915+105 as the most likely counterpart, assuming mass scaling of the QPO frequency. This is supported by the fact that these data from GRS $1915+105$ have an energy spectrum which is also dominated by a 'hot disc' component. Here we show that the underlying broad band power spectral shape and normalisation are also similar, providing further consistency checks for this identification. Thus the AGN QPO adds to the growing evidence for a simple mass scaling of the accretion flow properties between stellar and supermassive black holes.
\end{abstract}

Key words: accretion, accretion discs - X-rays: binaries, black hole

\section{INTRODUCTION}

Active galaxies (AGN) and accreting Black hole binaries (BHB) are the most energetic and dynamic systems in the Universe, with intrinsic variability seen over a broad range of timescales. This variability is most often quantified via a power spectrum, showing the (squared) amplitude of variability as a function of frequency. For BHB this spectrum can be very approximately described as band limited noise, with a 'flat top' in $\nu P(\nu)$ (equal variability power per decade in frequency) i.e. $P(\nu) \propto \nu^{-1}$. This extends between a low and high frequency break, $\nu_{b}$, below which the PDS is $P(\nu) \propto \nu^{0}$, and $\nu_{h}$, above which the spectrum steepens to $P(\nu) \propto \nu^{-2}$. While this is adequate to describe the limited statistics of AGN power spectra (see the compilation of Markowicz et al. 2003), the excellent data from BHB show that band limited noise is intrinsically bumpy, and is better represented by a series (generally $4-5$ ) of peaked noise components (Belloni \& Hasinger 1990; Psaltis, Belloni \& van der Klis 1999; Nowak 2000; Belloni, Psaltis \& van der Klis 2002). Superimposed on this continuum are a series of narrower features, referred to as Quasi-periodic oscillations (QPOs). These QPOs come in two distinct categories, termed low and high frequency QPOs respectively, and their properties depend strongly on mass accretion rate (see e.g. the reviews by van der Klis 2004; Remillard \& McClintock 2006; Done, Gierliński \& Kubota 2007, hereafter DGK07).
There is now considerable evidence to suggest that AGN and BHB are mass-scaled analogies and share the same underlying physics of accretion, notably the Radio/X-ray fundamental plane relations (Merloni, Heinz \& di Matteo 2003; Falcke, Körding \& Markoff 2004), variability scaling (McHardy et al. 2006; McHardy 2009) and mass-accretion rate dependant spectral behaviour (Pounds, Done \& Osborne 1995; Middleton, Done \& Schurch 2008; Middleton, Done \& Gierliński 2007). If this is the case then we would naturally expect QPOs to be visible in the power spectra of AGN at frequencies inversely scaled with the mass. However, these much longer timescales make detecting such QPOs in AGN very challenging (Vaughan \& Uttley 2006). Many claims for such periodic signals have been made, but these all failed to properly determine the significance of the QPO on top of the (uncertain determination) of the underlying red noise power spectrum (see the discussion in Vaughan 2005). By contrast, our discovery of a $\sim 1$ hour periodic signal from a Narrow Line Seyfert 1 (NLS1), REJ1034+396 is highly significant $(>99 \%)$ with correct modelling of the red noise spectrum (Gierliński et al. 2008). This gives the first detected AGN QPO to compare with the BHB. 


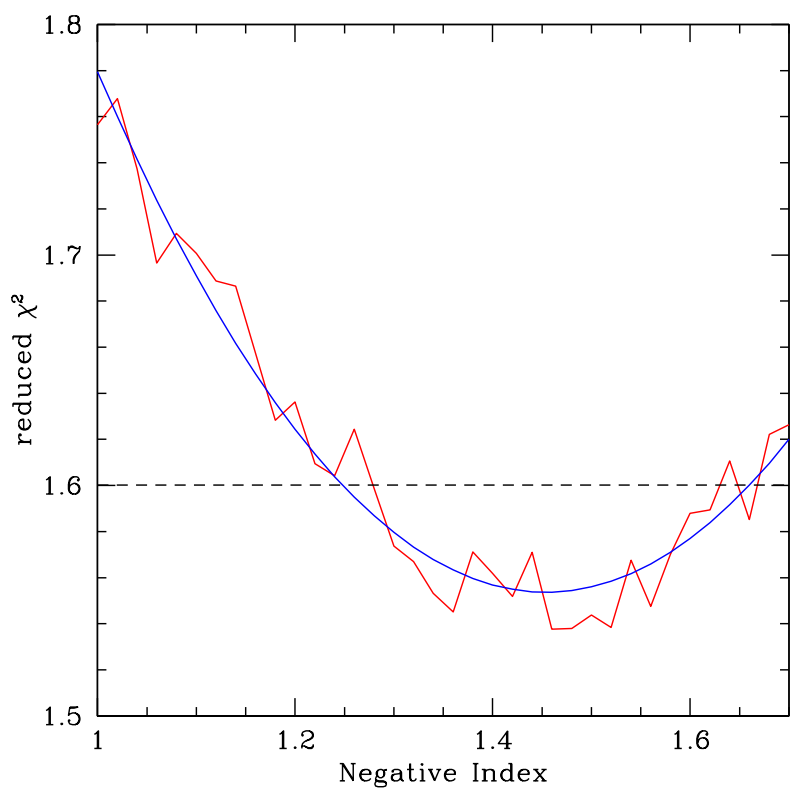

Figure 1. Reduced $\chi^{2}$ (58 d.o.f.) plot for single power-law power spectrum simulations for the 'in phase' lightcurve. We fit this to a quadratic function to smooth over the intrinsic stochastic effects. We use this to define the best fit index and its uncertainty $\left(\Delta \chi^{2}=2.7\right.$ i.e. $90 \%$ confidence as shown by the dashed horizontal line) as $-1.45_{-0.21}^{+0.20}$. This is consistent with the analytical result of $-1.35 \pm 0.18$ (Gierliński et al. 2008).

\section{MASS SCALING}

Beyond simply detecting the QPO, we need to identify it with one of the multiple BHB QPOs. Up until recently this has been a problem as the mass of the AGN black hole was not well constrained, with different techniques $(\mathrm{H} \beta$ line width, stellar velocity dispersion) giving mass estimates which differ by a factor of 30-50 (Gierliński et al. 2008). However, a recent reanalysis of the SDSS data from this object shows that both these black hole mass tracers can give consistent estimates for the black hole mass with careful decomposition of the different spectral components, giving $1-4 \times 10^{6} \mathrm{M}_{\odot}($ Bian \& Huang 2009). This predicts an analogous signal from a $10 \mathrm{M}_{\odot} \mathrm{BHB}$ between 27 and $108 \mathrm{~Hz}$ if a simple linear mass scaling holds for this QPO.

Placing this mass estimate together with the bolometric luminosity of $\sim 5 \times 10^{44} \mathrm{ergs} \mathrm{s}^{-1}$ implies $1<\mathrm{L} / \mathrm{L}_{E d d}<4$. This is supported by the distinctive nature of the optical/UV/Xray SED of REJ1034+396, where the bolometric luminosity is dominated by a very luminous component peaking in the EUV (Puchnarewicz et al. 2001; Middleton, Done \& Gierliński. 2007). This cannot be fit by standard disc models, but can be approximated by the super-Eddington slim disc models (Mineshige et al. 2000; Wang \& Netzer 2003, Haba et al. 2008).

The type and characteristic frequency of QPOs seen from BHB depend on mass accretion rate as well as BHB mass. Thus we need to study a super-Eddington BHB in order to find an analogue with the AGN QPO. GRS 1915+105 is the only BHB which spends significant time at (probably) super-Eddington accretion rates (Done, Wardzinski \& Gierliński 2004). This has a mass of $\sim 14 \mathrm{M}_{\odot}$ (Greiner, Cuby

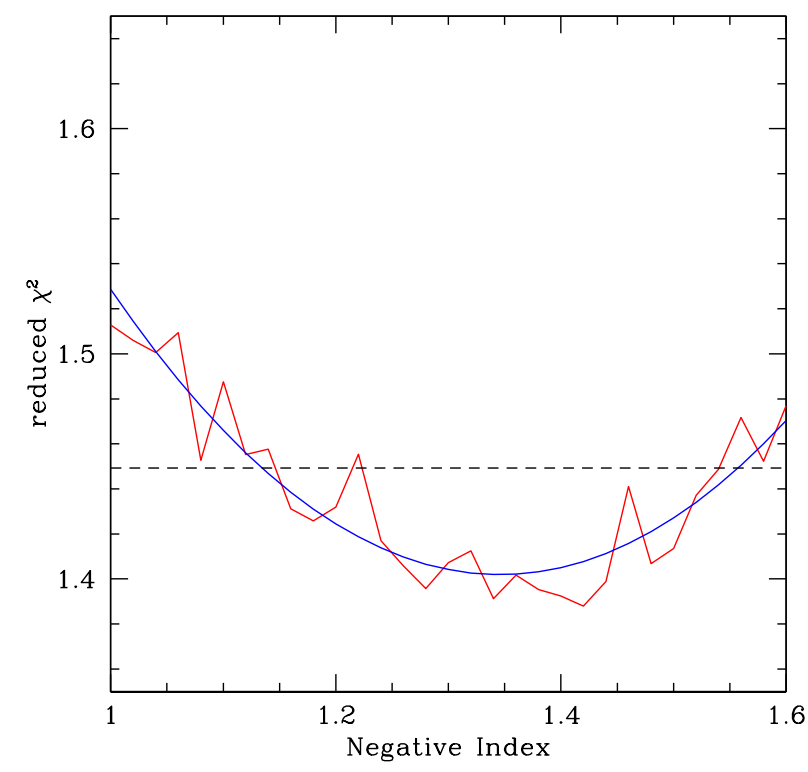

Figure 2. As for Fig 1. but excluding the single frequency bin of the QPO in the 'in phase' segment (57 d.o.f.). The result is consistent with that of simulations including the QPO over the same segment, as expected as the QPO is fairly central in the power spectrum so this power does not distort the index.

\& McCaughrean 2001; Harlaftis \& Greiner 2004), so a simple mass scaling of the AGN QPO predicts the counterpart is between 19 and $77 \mathrm{~Hz}$. GRS1915+105 does indeed occasionally show a QPO at $67 \mathrm{~Hz}$ (with a probable harmonic at $41 \mathrm{~Hz}$ ), making this the most likely candidate.

This means that the data are consistent with the simplest model, which is that the QPO seen at a given luminosity/spectral state in BHB scales linearly with mass up to AGN. However, this only makes use of the information on the QPO frequency. This QPO sits on top of an underlying broad band continuum noise power spectrum, and the shape and normalisation of this broadband noise is likewise dependent on luminosity/spectral state in BHB. Here we use the additional information from this noise power to check the QPO identification.

\section{POWER SPECTRAL PHENOMENOLOGY IN BHB}

\subsection{Low-frequency QPOs}

These are subdivided into 3 classes, types A, B and C (see e.g. Casella et al. 2005), not to be confused with the spectral states in GRS 1915+105 which are given the same name (Belloni et al. 1997)!

The C class LFQPO is almost always present in the low/hard state of BHB. It has frequency which increases between $\sim 0.01-6 \mathrm{~Hz}$ as the spectrum softens from a dim, harder, low/hard state to a bright, softer low/hard state, through to the hard intermediate state (Belloni et al. 2005). There is a correlated increase in strength, coherence and harmonic structure of the QPO (van der Klis 2004; Remillard \& McClintock 2006). Its spectrum closely follows that of the harder X-ray Comptonisation component rather than 
the soft disc emission (Rodriguez et al. 2002; Sobolewska \& Zycki 2003; 2006), so it is most probably produced by some fundamental mode of the corona, not of the disc.

The changes in underlying power spectral shape are correlated with the energy spectral changes and the QPO frequency changes. Very approximately, the type C QPO frequency increases together with the low frequency break, $\nu_{b}$, (Psaltis et al. 1999), while the high frequency break remains constant. Thus the broad band power spectrum progressively narrows, losing the low frequency power (Gierliński, Nikołajuk \& Czerny 2008). The normalisation of the 'flat top' remains roughly constant at $\nu P(\nu) \approx 0.01$ when the power spectrum is normalised to the fractional fluctuation power $(\sigma / I)^{2}$ at high energies. The bandpass is important as the disc component becomes progressively stronger as the spectrum softens. The disc remains mostly constant on the short timescales of the power spectrum, so dilutes the variability of the higher energy tail (Churazov et al. 2001; Done \& Gierliński 2005).

In the context of the truncated disc/hot inner flow models for the low/hard state, all these observations can be interpreted if the inner edge of the thin disc decreases as a function of mass accretion rate. The increasing overlap between the disc and hot flow means more seed photons from the disc intercept the hot flow, giving stronger Compton cooling and softer spectra. The moving inner radius means that the section of the hot flow which is unconstrained by the disc gets smaller, so increasing all its characteristic variability frequencies, while the smaller radial extent means the QPO can become stronger and more coherent (DKG07). This qualitative picture can be made quantitative, using vertical (Lense-Thirring) precession of the hot flow to make the QPO (Ingram, Done \& Fragile 2009), and turbulence in the hot flow to make the broadband power (Ingram \& Done 2009), but such models are still at the speculative stage.

The type B LFQPO is seen as the source continues to soften after the highest type C LFQPO from the hard intermediate state to the soft intermediate state. This is a rather subtle change in spectrum, but triggers an abrupt collapse of the underlying broad band noise power spectrum, leaving the QPO (and its multiple harmonics) dramatically enhanced in prominence (Remillard et al. 2002; Belloni et al. 2005).

As the spectrum continues to soften in the soft intermediate state, class A LFQPOs can be seen. These are weaker and much broader (so there is no harmonic structure discernible) than the type B LFQPOs, but appear at similar frequencies, and again are seen against a very low continuum noise power (Remillard et al. 2002). Then the QPOs disappear altogether as the spectrum continues to soften into a completely disc dominated state (Remillard \& McClintock 2006; Casella et al. 2005).

While the type C LFQPO is ubiquitous in the low/hard and hard intermediate states, types $\mathrm{B}$ and $\mathrm{A}$ are much rarer, seen only in the soft intermediate state.

\subsection{High-frequency QPOs}

Transient high-frequency QPOs (HFQPOs) are detected in several BHB sources (GRO J1655-40, XTE J1550-564, GRS 1915+105, 4U 1630-47 and XTE J1859+226). These appear to be constant in frequency despite changes in lumi-

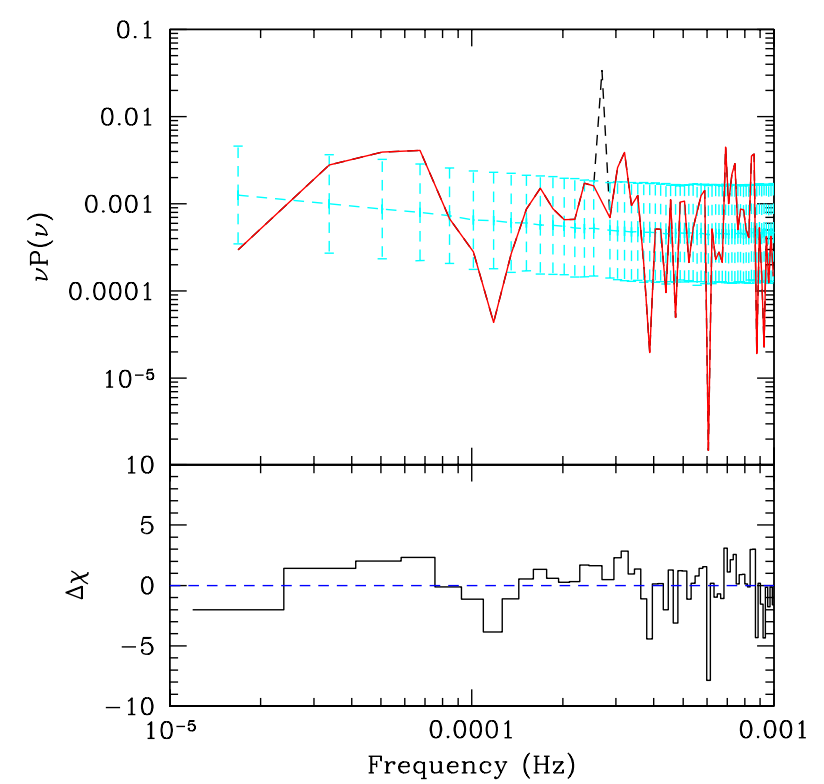

Figure 3. The upper panel shows the best-fit average simulated power spectrum (cyan) to that of the 'in phase' data (red) without the QPO (shown as the dashed black line). This assumes a single power-law form for the intrinsic fluctuations, together with statistical (white) noise. The dispersion in the simulations is shown by the dashed error bars (cyan). The lower panel shows residuals to this best fit.

nosity by a factor of $3-8$, so these may be a stable signature of the accretion system. The values are consistent with being directly proportional to the mass of the black hole, though there are only 3 data points and the masses only range from $6-14 M_{\odot}$ (Remillard \& McClintock 2006). They are often seen in 3:2 ratio, implying some sort of resonance sets the frequencies, but the exact nature of this resonance is yet to be clearly determined (Abramowicz \& Kluzniak 2001; Kluzniak et al. 2004; Török et al. 2005; Kato et al. 2004; Rezzolla et al. 2003; Fragile et al. 2005).

These seem to be detected only in the soft intermediate state i.e. are seen simultaneously with type B and A LFQPOs and not with type C LFQPOs (Remillard et al. 2002). It is extremely difficult to accurately assess the underlying noise power spectral shape at such high frequencies as this is dominated by the statistical white noise, but the coadded spectra of Remillard et al. (2002) suggest that these high frequency QPOs sit on a broad band continuum which has a $\nu^{-1}$ shape (most probably another Lorentzian component) but with a normalisation which is over an order of magnitude below that seen for the lower frequency 'flat top' noise which accompanies the type C LFQPOs (Remillard et al. 2002). While the normalisation of the power spectrum strongly depends on the energy bandpass in BHB due to the different variability of disc and Comptonisation components (e.g. Churazov et al. 2001; Done \& Gierliński 2005), the study of Remillard et al. (2002) used only data above $6 \mathrm{keV}$ where the disc makes little contribution. Hence this difference in normalisation is probably intrinsic rather than due to dilution of the variability by the constant disc. 


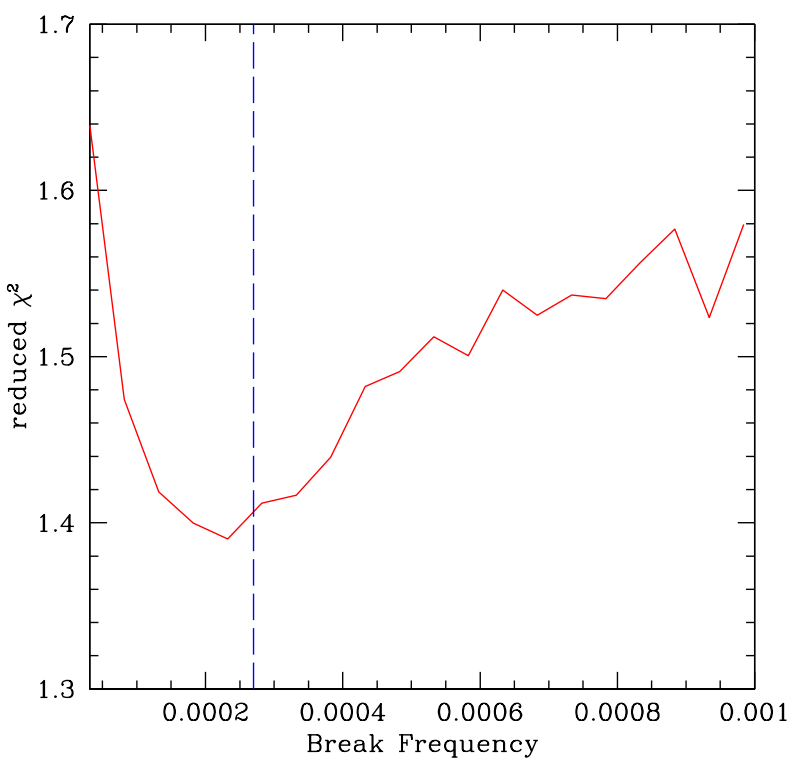

Figure 4. As in Fig 1 but for an underlying broken power-law (indices from -1 to -2) spectral shape of the 'in phase' segment of the lightcurve without the QPO. The $\chi^{2}$ of the best fit of a break close to the QPO frequency $\left(2.7 \times 10^{-} 4 \mathrm{~Hz}\right.$ : vertical dashed line) is similar to that for the single power-law fit in Fig 2, so this is not a significantly better description of the spectral shape.

\subsection{Additional QPOs seen only in GRS 1915+105}

GRS $1915+105$ is highly luminous, so never gets to the low/hard state. Instead it ranges between the hard intermediate state, soft intermediate state and disc dominated states. However, it also shows spectra which are unlike any of the standard states, being dominated by low temperature Comptonisation of the disc together with a very weak hard tail (Zdziarski et al. 2003; Done, Wardzinski \& Gierlinski 2004; Belloni 2009). In this state it can show some particularly unique QPOs, including very strong, narrow QPOs with complex harmonic structure at tens of milliHertz (Morgan, Remillard \& Greiner 1997). These are startlingly similar to the QPO frequencies seen in the Ultra-Luminous Xray sources M82 X-1 (Strohmayer \& Mushotzky 2003) and NGC5408 X-1 (Strohmayer at al. 2007). While these low frequencies clearly imply intermediate mass black holes if they are identified with the standard LFQPO, it is plain from the data of GRS 1915+105 that such low frequencies can also be formed from super-Eddington accretion onto a $14 M_{\odot}$ black hole (Heil, Vaughan \& Roberts 2009). Simultaneously with this very low frequency QPO, GRS $1915+105$ can also show an occasional QPO at $67 \mathrm{~Hz}$. This has only been strongly detected in a few datasets (Morgan, Remilllard \& Greiner 1997) plus a few other weaker detections (Morgan, Remilllard \& Greiner 1997; Ueda et al. 2009), together with a (potential harmonic) feature at $41 \mathrm{~Hz}$ (McClintock \& Remillard 2006). The statistical noise is not so dominant as for the HFQPOs as these are at lower frequencies, so the underlying continuum noise power is somewhat easier to constrain. This has a complex shape, but the normalisation underneath the QPO appears to be $\nu P(\nu) \sim 10^{-4}$ from Morgan et al. (1997). However, this used power spectra across the full en-

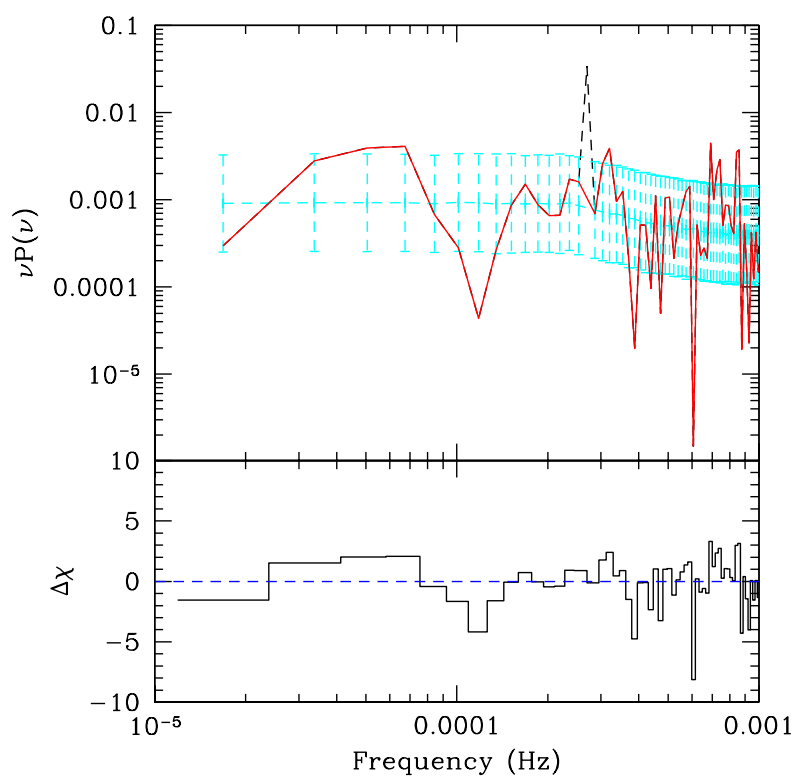

Figure 5. As in Fig 3 but for a broken power-law (indices from -1 to -2$)$ fit to the 'in phase' power spectrum without the QPO.

ergy bandpass. Concentrating instead on the high energy band gives $\nu P(\nu) \sim 0.001$ (Remillard \& McClintock 2006).

\section{LIGHTCURVE SIMULATIONS}

The analytical method of fitting the broadband shape of the power spectrum of Vaughan (2005) is only applicable to a single power-law continuum noise shape, so does not include intrinsic breaks or the effects of statistics (white noise at high frequencies). Instead we simulate an ensemble of lightcurves with given power spectral properties using the method of Done et al. (1992), using the corrected power spectral statistics (Timmer \& Koenig 1995), as developed by Uttley et al. (2002).

The real data are evenly sampled and binned on timescale $\Delta t$ and span a total time of $T_{\text {data }}$. This has mean, $I$, total variance, $\sigma_{\text {data }}^{2}=\sigma_{\text {int }}^{2}+\sigma_{\text {err }}^{2}$, where $\sigma_{\text {int }}^{2}$ is due to the intrinsic variability of the source and $\sigma_{e r r}^{2}$ is the additional variance added by statistical uncertainties. We generate a lightcurve using the fast Fourier transform method of Timmer \& Koenig (1995), spanning a timescale $\mathrm{N} T_{\text {data }}$ (where $\mathrm{N} \gg 1$ ) with some intrinsic power spectral shape, $P(f) \sin ^{2}(2 \pi f \Delta t) / 2 \pi f \Delta t$, where the $\sin ^{2}$ term accounts for the suppression of high frequency power due to binning (Done et al. 1992). We snip this up into $\mathrm{N}$ separate lightcurves of length $T_{\text {data }}$. For each lightcurve we measure $\sigma_{i, i n t}^{2}$ and $I_{i}$, and then scale so that the average mean and variance of the ensemble is the same as that in the real data. We then add white noise with the same properties as in the real data, take the power spectra of the simulated lightcurves and use these to define the statistics of the power spectral distribution.

One key problem with this method is that it is linear while the real data are known to be non-linear. The physical process which generates the noise keeps $\sigma_{i n t} / I$ con- 


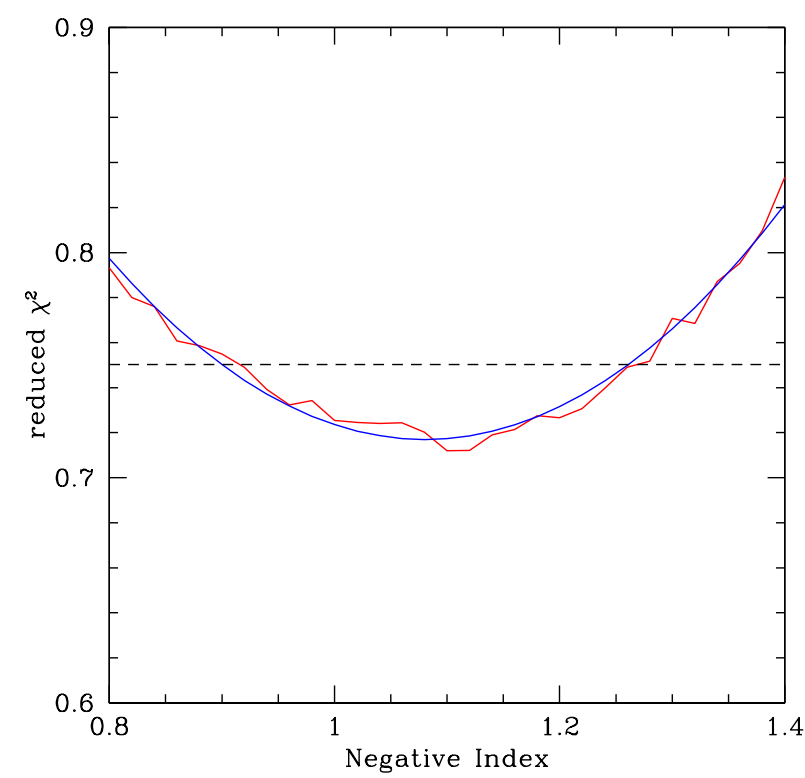

Figure 6. As in Fig 1 for a single power-law description of the power spectrum without the QPO from the full lightcurve (81 d.o.f.)

stant as the source varies (Uttley \& McHardy 2001). This is quite unlike the simulated lightcurve properties e.g. for $P(f) \propto \nu^{-\alpha}$ with $\alpha>1$ (red noise) there is no well defined mean, so no well defined $\sigma_{i, i n t} / I_{i}$. Thus the snipped lightcurves made from this power spectrum have large dispersion in $\sigma_{\text {int }} / I$, so a large range in power spectral normalisation, defined as $\int P(f) d f=\left(\sigma_{\text {int }} / I\right)^{2}$. This wide dispersion is unlike that of the real data, where the noise process is self-similar. An exponential transform of the simulated linear lightcurve can recover approximately the required statistical properties (Uttley, McHardy, \& Vaughan 2005) but instead we simply choose to put a break in the input power spectrum at $0.5 / T_{\text {data }}$. At frequencies below the break the spectrum is flat $(\alpha=0)$ and above the break the spectrum follows the desired input power spectral shape. The result is a set of snipped lightcurves of the same length and statistical properties as the real data, with a well defined mean and dispersion.

\section{THE UNDERLYING CONTINUUM POWER SPECTRAL SHAPE OF REJ1034+396}

XMM-Newton observed REJ1034+396 on 2007-05-31 and 2007-06-01 for $\sim 91 \mathrm{ks}$ (observation id. 0506440101, revolution no. 1369). Details of the data extraction are given in Middleton et al. (2009). This resulted in $84.3 \mathrm{ks}$ of clean data starting on 2007-05-31 20:10:12 UTC. The QPO is very coherent over 16 periods in a $59 \mathrm{ks}$ segment of this total lightcurve, hereafter called the 'in phase' data. The resulting power spectrum of this 'in phase' data is shown in figure 3, where the QPO is present at $>99.9 \%$ significance above the red noise (Gierlińksi et al. 2008). The index of the total (i.e. including the QPO) power spectrum over the frequency range not dominated by statistical noise (i.e.

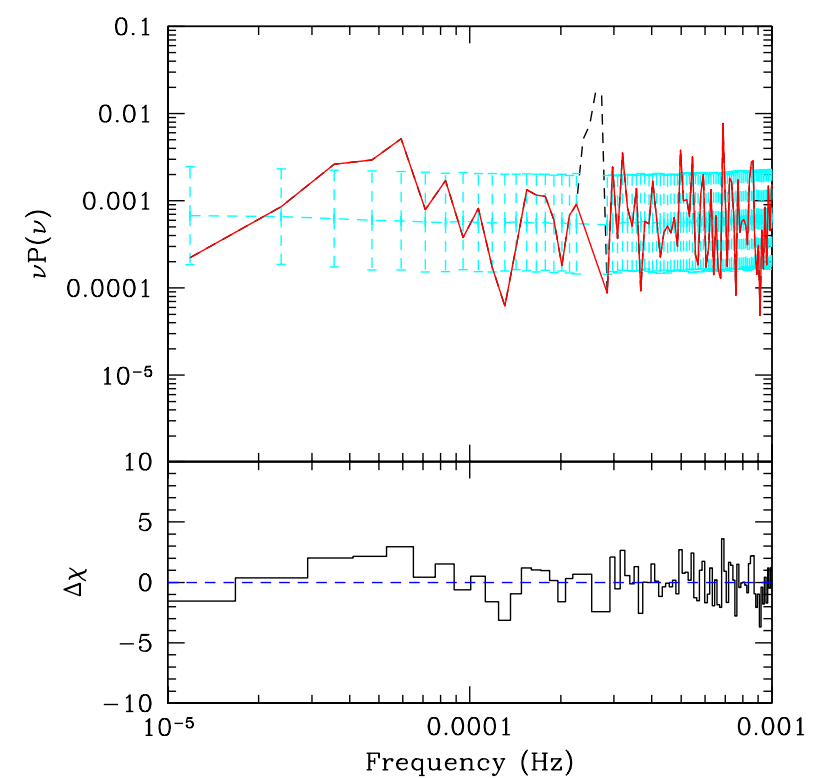

Figure 7. As in Fig 3 for the best-fit single power-law fit to the power spectrum without the QPO from the full lightcurve.

above $10^{-3} \mathrm{~Hz}$ ) was estimated using the analytical method of Vaughan (2005) to be $-1.35 \pm 0.18$ (Gierliński et al. 2008)

\subsection{Simulations of the in-phase segment}

We first check that our simulations reproduce the analytical result. Fig 1 shows the $\chi^{2}$ versus power spectral index for a single power-law fit to the entire power spectrum above $10^{-3} \mathrm{~Hz}$. The simulations are inherently stochastic so there are fluctuations. We smooth these by fitting a quadratic which gives a minimum at $-1.45{ }_{-0.21}^{+0.20}$, consistent with the analytical result.

However, here we are more interested in the shape of the power spectrum which underlies the QPO, so we remove the single point at the QPO frequency, replacing it with the fractional rms of the bin directly preceding it. We then redo the single power-law simulations with this reduced $\left(\sigma_{\text {int }} / I\right)^{2}$. We show the results from this in Fig 2, again fitted with a quadratic to smooth over the inherent stochasticity. This gives $-1.35_{-0.21}^{+0.22}$ similar to the previous result as the QPO is fairly central in the fitted section of the power spectrum, so does not greatly distort the average power spectral slope. The fit is obviously better in terms of overall reduced $\chi^{2}$, and is an acceptable description of the data at $\sim 97 \%$ confidence. This best-fit average power spectrum is shown together with residuals in Fig 3.

We repeat the analysis using a broken power-law, fixing the slope below and above the break to -1 and -2 respectively, and then varying the break frequency. Fig 4 shows that there is a minimum in $\chi^{2}$ for such a break at a frequency close to the QPO. However, the value of $\chi^{2}$ is slightly (though not significantly) higher than that for a single power-law description of the underlying power spectrum, so this is not a better description of the power spectrum than a single power-law. The best-fit, average broken power-law together with errorbars from statistical uncertainties is shown in fig- 


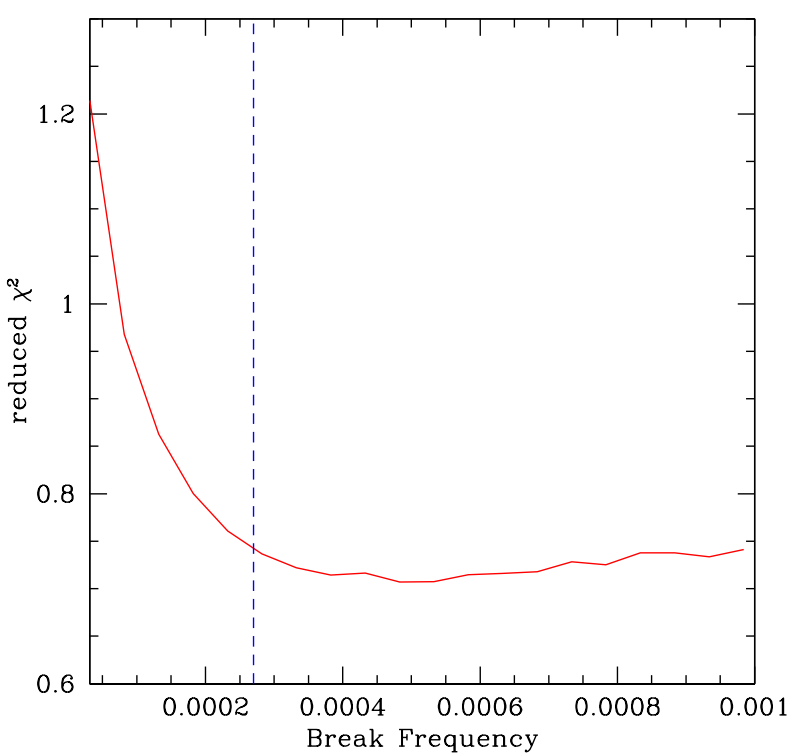

Figure 8. As in Fig 1 for simulations of a broken power-law (indices -1 to -2 ) fit to the power spectrum without the QPO from the full lightcurve. Again, this is not a significantly better fit than the single power-law shown in Fig 6.

ure 5 together with the residuals to the real power spectrum (minus the QPO) in the panel below.

\section{$5.2 \quad$ Full lightcurve}

The longer timescale spanned by the 'full' lightcurve extends the power spectrum down to slightly lower frequencies so may give tighter constraints on any power spectral break. However, the QPO is now not so coherent, so is spread over more frequency bins. We simulate the underlying power spectrum by excluding 5 frequency bins around the QPO frequency, and replacing them with the preceding bins. Figure 6 shows the resulting $\chi^{2}$ distribution for the single power-law, which has index $-1.08 \pm_{-0.18}^{+0.18}$. The overall reduced $\chi^{2} \approx 1$, showing that this is a good description of the data. Figure 7 shows the best-fit average single power-law fit to the real power spectrum. Error bars come from statistical uncertainties and, as before, the residuals to the fit are shown in the panel below.

We again test for the presence of a break in the underlying red noise (excluding the QPO) by simulating a broken power-law with indices fixed at -1 and -2 . The resulting chisquared plot (figure 8) again shows that such a spectrum with break frequency slightly above the QPO is consistent with the data, but is not required by it. As before, the bestfit broken power-law is plotted in figure 9 .

\subsection{Summary of constraints from the power spectral shape}

The underlying power spectral shape is consistent with a single power-law with index -1.3. This is inconsistent with the $\sim \nu^{-2}$ shape seen at high frequencies in the sub-Eddington states of Cyg X-1 (Revnivtsev et al. 2000), but may be consistent with the (poorly constrained) noise power shape un-

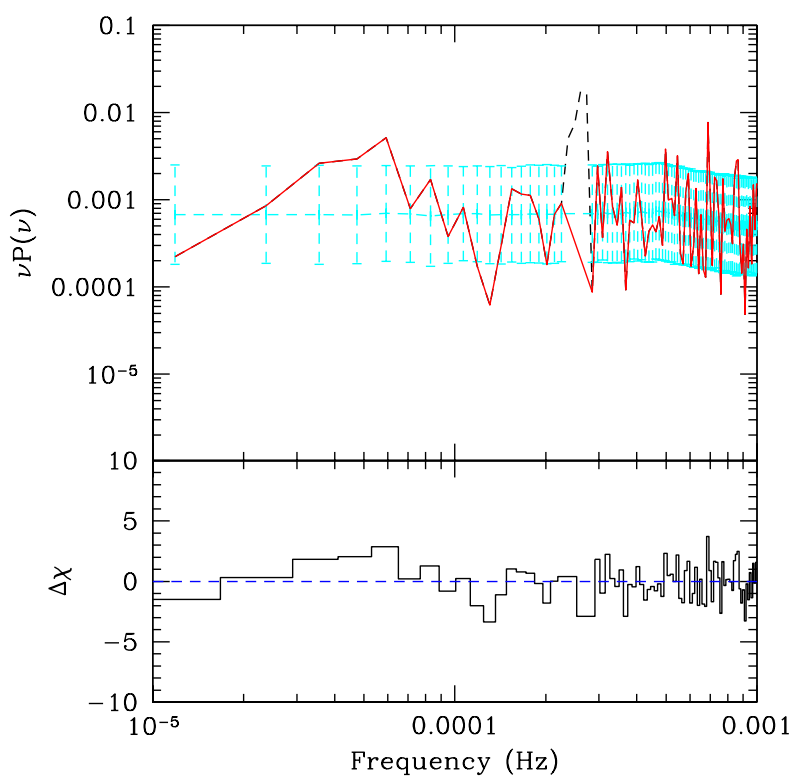

Figure 9. As in Fig 3 for the best fit broken power-law (indices -1 to -2$)$ fit to the power spectrum without the QPO from the full lightcurve.

derlying the HFQPO or $67 \mathrm{~Hz}$ QPO. While this supports our tentative identification of the QPO with the $67 \mathrm{~Hz}$ feature, the power spectral shape is also consistent with that expected underneath the LFQPO, i.e. with a power spectrum which breaks from $\nu^{-1}$ to $\nu^{-2}$ at a frequency just above the LFQPO frequency. However, this can be ruled out by the power spectral normalisation, which is an order of magnitude below the flat-top normalisation of $\nu P(\nu) \sim 0.01$ seen ubiquitously under the LFQPO. Thus the power spectrum normalisation precludes an identification with the LFQPO. This supports the conclusions from the source spectrum and luminosity, which favour a mildly super-Eddington accretion flow, whereas the LFQPOs are characteristic of subEddington states.

Instead, the power spectral normalisation is similar to that seen from the HFQPOs and the $67 \mathrm{~Hz}$ QPO from GRS 1915+105. However, the HFQPOs are again seen from sub-Eddington states, so the $67 \mathrm{~Hz}$ QPO from GRS1915+105 is our most likely candidate (see Section 2). Thus we explore the power spectral correspondence in more detail below.

\section{ENERGY SPECTRA}

Bandpass effects are very important, as different components have different variability, and this can substantially affect the derived normalisation of the power (Churazov et al. 2001; Done \& Gierliński 2005). Hence we now look in detail at the energy spectra for GRS $1915+105$ at the time when it shows the $67 \mathrm{~Hz}$ QPO, our most likely candidate for the AGN QPO, and compare components and bandpass with REJ1034+396.

The data from GRS 1915+105 which show the $67 \mathrm{~Hz}$ QPO are the RXTE observations on April the 20th (OBSID: 10408-01-04-00), April the 29th (OBSID: 10408-01-05- 


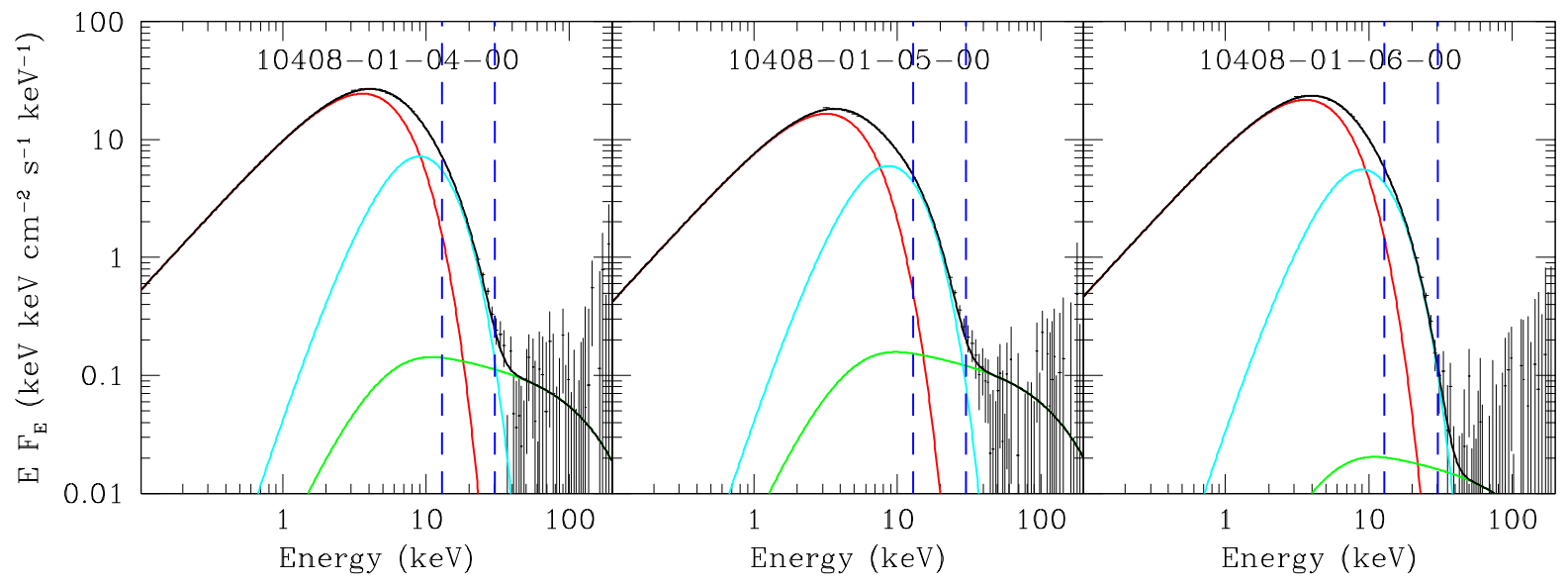

Figure 10. Unabsorbed PCA and HEXTE X-ray spectra of GRS $1915+105$ in the three observations showing the $67 \mathrm{~Hz}$ QPO at $>3 \sigma$. The total model (black) is made of components from a disc blackbody (red), low temperature, optically thick thermal Comptonisation (cyan), and thermal Comptonisation with a hotter plasma (green). The vertical dashed lines indicate the 13-30 keV energy band used to create the power spectrum shown in Fig $12 \mathrm{~b}$

00) and May 5th (OBSID: 10408-01-06-00) 1996 (Morgan, Remillard \& Greiner 1997). The energy spectra of these observations are shown in Fig 10, fit with a disc black-body component (DISKBB) and a low-temperature, optically thick thermal Compton component (COMPTT). This is accompanied by a tail to higher energies, which we model as high temperature thermal Comptonisation, with power-law index fixed at 2.2 and electron temperature at $100 \mathrm{keV}$. The tail is probably much more complex (see e.g Zdziarski et al. 2005), but this gives an adequate description of the $R X T E$ data. We show the data with its corresponding best fit model, corrected for absorption (fixed to the variable abundance model used in Done, Wardzinski \& Gierliński 2004), superimposed on the data in Fig 10.

Fig 11 shows the spectrum of REJ1034+396 fit by the same three component model. This has a ratio of inferred (bolometric) disc flux to Comptonised flux of 3.0, and a ratio of disc to power-law flux of 19 , similar to that of GRS $1915+105(4.3 / 38,3.0 / 24$ and $4.3 / 33$ for the 04,05 and 06 ObsIDs respectively). However, the ratio of electron temperature to disc temperature is rather higher for REJ1034+396 than for GRS 1915+105 (6.6 versus 1.6, 1.8 and 1.7), making the approximately power-law section of the 'soft excess' rather than Wien shape of this component. There are other energy spectra in GRS 1915+105 which match this aspect more closely (Middleton et al. 2009), but these data do not show a significant high frequency QPO.

We can now quantify the bandpass effects. In GRS 1915+105, Remillard et al. (2004) show the $41 / 67 \mathrm{~Hz}$ QPO computed from the lightcurve in the $13-30 \mathrm{keV}$ bandpass range, rather than the $2-20 \mathrm{keV}$ in Morgan et al (1997). We overlay this energy range on the spectra in Fig 10 to show that this samples mostly the low temperature Comptonisation component, excluding most of the disc component which dilutes the variability, as well as most of the higher energy power-law. To be dominated by the corresponding spectral components in REJ1034+396 requires a bandpass of $0.3-1.5 \mathrm{keV}$.

Fig $12 \mathrm{a}$ shows the resulting power spectrum from 0.3 -

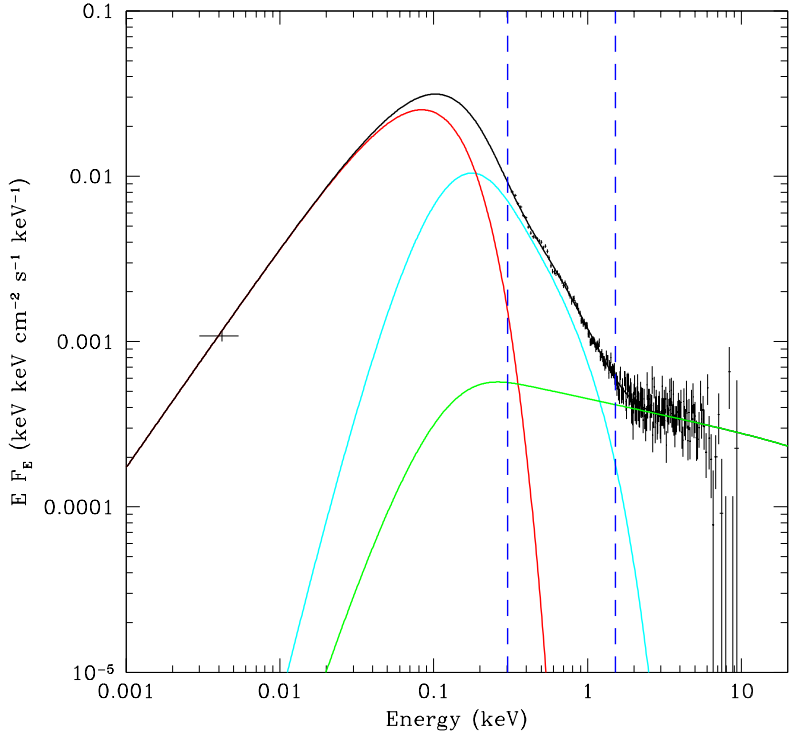

Figure

11.

Unabsorbed Optical/UV/X-ray SED of REJ1034+396 showing the deconvolved disc (red), low-temperature Compton component (cyan) and Comptonisation from a higher temperature plasma (green). The vertical dashed lines indicate the energy band which contains a similar amount of the spectral components (dominated by the low temperature Comptonisation) as in the $13-30 \mathrm{keV}$ energy band in GRS 1915+105 (Fig 10).

$1.5 \mathrm{keV}$. Unlike all the other power spectra, this one has statistical noise subtracted, so has less power at high frequencies. However, the intrinsic power is very similar to that of the total lightcurve in both shape and normalisation. Fig $12 \mathrm{~b}$ shows the 13-30 keV power spectrum of GRS $1915+105$ of Remillard et al. (2004), again with statistical noise subtracted. This is plotted using the same scale in $\nu P(\nu)$ over 1.5 decades in frequency $(10-300 \mathrm{~Hz}$ in GRS $1915+105$, scaled by the putative mass difference of $3 \times 10^{6} / 14$ corresponding 


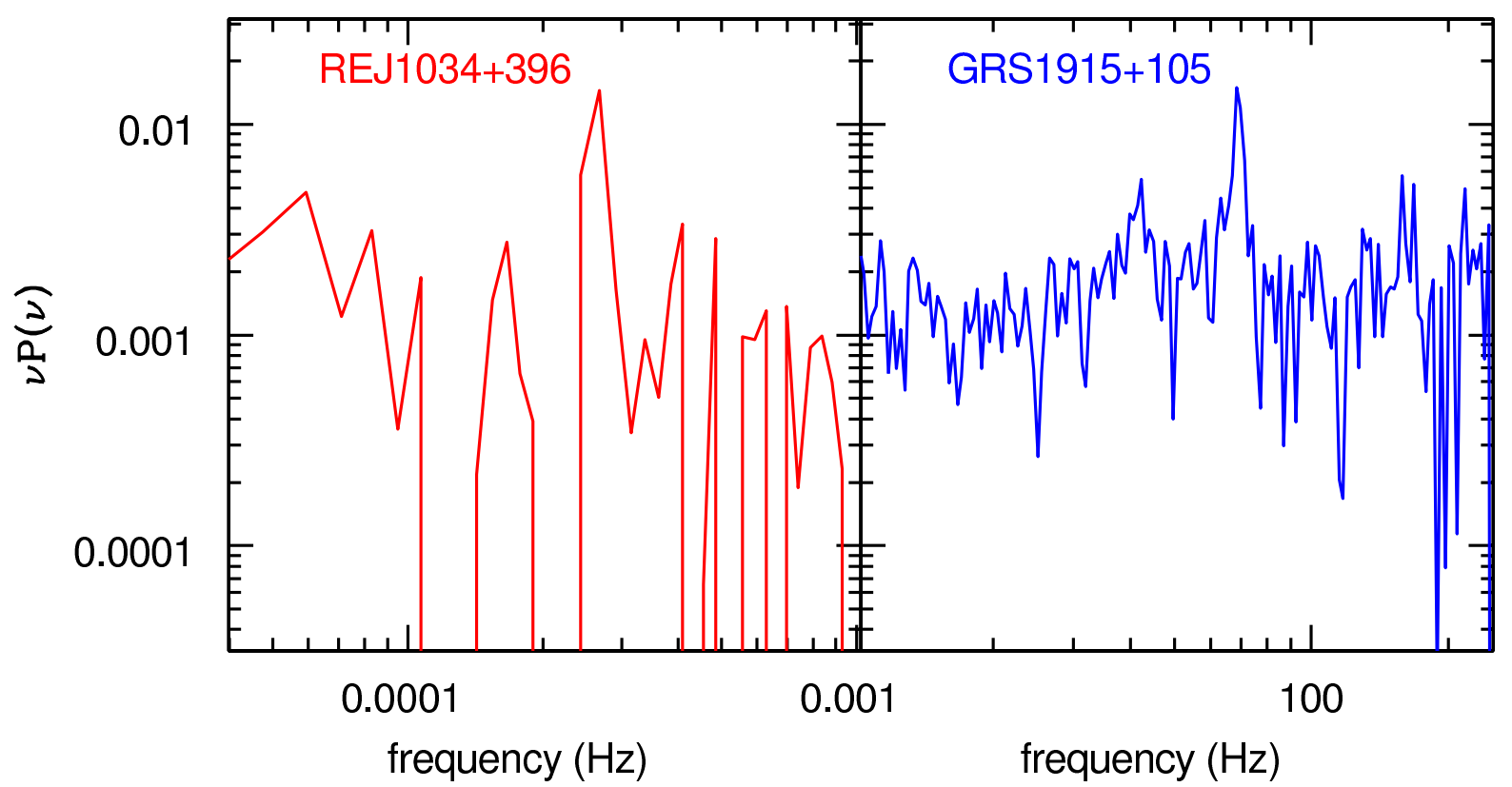

Figure 12. (a) shows the PDS of REJ1034+396 in the 0.3-1.5keV energy band, while (b) shows the corresponding energy bandpass 13-30 keV PDS from GRS 1915+105 (Remillard et al. 2003). Unlike the other power spectra shown in this paper, the statistical noise has been subtracted from both, so that the PDS shows only the intrinsic power. Both cover 1.5 decades in frequency, and are on the same scale. Both normalisation and slope underlying the QPO are very similar, supporting the identification of the AGN QPO as analogous to the $67 \mathrm{~Hz}$ QPO in GRS 1915+105.

to $4.66 \times 10^{-5}-1.4 \times 10^{-3} \mathrm{~Hz}$ in REJ1034+396). While the power spectrum of the AGN has very poor statistics compared to GRS $1915+105$, these data are startlingly similar in both shape and normalisation for both the QPO and underlying power spectrum. Much better data would be required in order to constrain the existence of any 3:2 harmonic corresponding to the $41 \mathrm{~Hz}$ QPO in GRS 1915+105 (it is barely significant in GRS 1915+105!). The slight excess of power at this frequency in the AGN is not at all significant.

\section{CONCLUSION}

The discovery of an AGN QPO allows us to test whether the physics of the accretion flow scales linearly with black hole mass if we can identify the corresponding BHB QPO. We use the variability power spectral shape and normalisation to show that the continuum noise underlying the AGN QPO is consistent with that seen underneath the HFQPO seen in BHB and $67 \mathrm{~Hz}$ QPO seen in GRS1915+105, but not with the LFQPO.

The distinctive shape of the energy spectrum of REJ1034+396 argues strongly for a mildly super-Eddington accretion flow in this AGN, supported by recent mass estimates for the black hole (Bian \& Huang 2009). This favours the $67 \mathrm{~Hz}$ QPO as the BHB counterpart, as this is seen in super-Eddington states of GRS $1915+105$, whereas the HFQPOs are seen in sub-Eddington states. The spectra seen from the $67 \mathrm{~Hz}$ QPO observations of GRS 1915+105 are similar to that the REJ1034+396 in being dominated by low temperature, optically thick Comptonisation of the disc. This spectral shape may well be the new 'ultra-luminous state' proposed by Gladstone et al. (2009) for the broad curvature seen in the X-ray spectra of the Ultra-Luminous $\mathrm{X}$-ray sources. Conversely, the disc is seen without strong distortion by Comptonisation in the spectra corresponding to the HFQPOs.

The identification of the AGN QPO at $2.7 \times 10^{-4} \mathrm{~Hz}$ with the $67 \mathrm{~Hz}$ QPO in GRS $1915+105$ is consistent with a simple linear mass scaling of the QPO frequency, energy spectral components and variability power spectra from stellar to supermassive black holes accreting at the same $L / L_{E d d}$.

\section{ACKNOWLEDGEMENTS}

We would like to thank NASA and ESA for making the data available for RXTE and XMM-Newton via the HEASRC website. This work was carried out through an STFC PhD grant. We would also like to thank Ron Remillard at MIT for helpful discussions and providing the data for the power spectrum of GRS $1915+105$ in figure 12 .

\section{REFERENCES}

Abramowicz M. A., Kluźniak W., 2001, A\&A, 374, L19

Belloni, T. M. 2009, arXiv:0909.2474

Belloni T., Hasinger G., 1990, A\&A, 230, 103

Belloni T., Mendez M., King A. R., van der Klis M., van Paradijs J., 1997, ApJ, 488, L109

Belloni T., Psaltis D., van der Klis M., 2002, ApJ, 572, 392

Belloni T., Méndez M., Homan J., 2005, A\&A, 437, 209

Bian, W.-H., \& Huang, K. 2009, arXiv:0909.1078 
Casebeer D. A., Leighly K. M., Baron E., 2006, ApJ, 637, 157 Casella P., Belloni T., Stella L., 2005, ApJ, 629, 403

Churazov E., Gilfanov M., Revnivtsev M., 2001, MNRAS, 321, 759

Done C., Gierliński M., 2005, MNRAS, 364, 208

Done C., Gierliński M., Kubota A., 2007, A\&ARv, 15, 1

Done C., Madejski G. M., Mushotzky R. F., Turner T. J., Koyama K., Kunieda H., 1992, ApJ, 400, 138

Done C., Wardziński G., Gierliński M., 2004, MNRAS, 349, 393

Falcke H., Körding E., Markoff S., 2004, A\&A, 414, 895

Fragile P. C., Miller W. A., Vandernoot E., 2005, ApJ, 635, 157

Gierliński M., Nikołajuk M., Czerny B., 2008, MNRAS, 383, 741

Gierliński M., Middleton M., Ward M., Done C., 2008, Natur, 455,369

Gladstone J. C., Roberts T. P., Done C., 2009, MNRAS, 948

Greiner, J., Cuby, J. G., \& McCaughrean, M. J. 2001, nat, 414, 522

Haba Y., Terashima Y., Kunieda H., Ohsuga K., 2008, AdSpR, 41,174

Harlaftis, E. T., \& Greiner, J. 2004, aap, 414, L13

Heil L. M., Vaughan S., Roberts T. P., 2009, MNRAS, 397, 1061

Ingram, A., \& Done, C. 2009, arXiv:0907.5485

Ingram, A., Done, C., \& Fragile, P. C. 2009, mnras, 397, L101

Kato Y., 2004, PASJ, 56, 931

Kluźniak W., Abramowicz M. A., Lee W. H., 2004, AIPC, 714 379

Markowitz A., et al., 2003, ApJ, 593, 96

McClintock J. E., Remillard R. A., 2006, csxs.book, 157

McHardy, I. M. 2009, arXiv:0909.2579

McHardy I. M., Koerding E., Knigge C., Uttley P., Fender R. P., 2006, Natur, 444, 730

Merloni A., Heinz S., di Matteo T., 2003, MNRAS, 345, 1057

Middleton M., Done C., Gierliński M., 2007, MNRAS, 381, 1426

Middleton M., Done C., Schurch N., 2008, MNRAS, 383, 1501

Middleton M., Done C., Ward M., Gierliński M., Schurch N., 2009, MNRAS, 394, 250

Mineshige S., Kawaguchi T., Takeuchi M., Hayashida K., 2000, PASJ, 52, 499

Morgan E. H., Remillard R. A., Greiner J., 1997, ApJ, 482, 993

Nowak M. A., 2000, MNRAS, 318, 361

Pounds K. A., Done C., Osborne J. P., 1995, MNRAS, 277, L5

Psaltis D., Belloni T., van der Klis M., 1999, ApJ, 520, 262

Psaltis D., Norman C., 2000, astro, arXiv:astro-ph/0001391

Puchnarewicz E. M., Mason K. O., Siemiginowska A., Fruscione A., Comastri A., Fiore F., Cagnoni I., 2001, ApJ, 550, 644

Remillard R., Muno M., McClintock J. E., Orosz J., 2003, nvm..conf, 57

Remillard R. A., Gazak J. Z., Lin D., Morgan E. H., Pooley G. G., 2004, AAS, 36, 1516

Remillard R. A., McClintock J. E., 2006, ARA\&A, 44, 49

Remillard R. A., Muno M. P., McClintock J. E., Orosz J. A., 2002, ApJ, 580, 1030

Revnivtsev M., Gilfanov M., Churazov E., 2000, A\&A, 363, 1013

Rezzolla L., Yoshida S., Maccarone T. J., Zanotti O., 2003, MNRAS, 344, L37

Rodriguez J., Durouchoux P., Mirabel I. F., Ueda Y., Tagger M., Yamaoka K., 2002, A\&A, 386, 271

Sobolewska M. A., Życki P. T., 2003, A\&A, 400, 553

Sobolewska M. A., Życki P. T., 2006, MNRAS, 370, 405

Strohmayer T. E., Mushotzky R. F., 2003, ApJ, 586, L61

Strohmayer T. E., Mushotzky R. F., Winter L., Soria R., Uttley P., Cropper M., 2007, ApJ, 660, 580

Timmer J., Koenig M., 1995, A\&A, 300, 707

Török G., Abramowicz M. A., Kluźniak W., Stuchlík Z., 2005, A\&A, 436, 1

Ueda Y., Yamaoka K., Remillard R., 2009, ApJ, 695, 888

Uttley P., McHardy I. M., 2001, MNRAS, 323, L26

Uttley P., McHardy I. M., Vaughan S., 2005, MNRAS, 359, 345
Uttley P., McHardy I. M., Papadakis I. E., 2002, MNRAS, 332, 231

van der Klis M., 2004, astro, arXiv:astro-ph/0410551

Vaughan S., 2005, A\&A, 431, 391

Vaughan S., Uttley P., 2006, AdSpR, 38, 1405

Wang J.-M., Netzer H., 2003, A\&A, 398, 927

Zdziarski A. A., Lubiński P., Gilfanov M., Revnivtsev M., 2003, MNRAS, 342, 355

Zdziarski A. A., Gierliński M., Rao A. R., Vadawale S. V., Mikołajewska J., 2005, MNRAS, 360, 825 